\title{
Humanity in Science Fiction Movies: A Comparative Analysis of Wandering Earth, The Martian and Interstellar
}

Ma Xinyi ${ }^{1 *}$ and Hua Jing ${ }^{2}$

${ }^{1} B A$ student, School of Foreign Languages, East China University of Science and Technology, Shanghai, China, 200237

${ }^{2}$ Associate Professor, School of Foreign Languages, East China University of Science and Technology, Shanghai, China, 200237,

Research interests: Comparative literature and culture

Corresponding Author: Hua Jing, E-mail: huajing0909@163.com

\section{ARTICLE INFORMATION ABSTRACT}

Received: December 01, 2020

Accepted: January 17, 2021

Volume: 4

Issue: 1

DOI: 10.32996/ijlt.2021.4.1.20

\section{KEYWORDS}

Science fiction movies; returning home; female role; intercultural communication
Wandering Earth, released in 2019, is regarded as a phenomenal film that opens the door to Chinese science fiction movies. The Chinese story in the film has aroused the resonance of domestic audiences, but failed to get high marks on foreign film review websites. In contrast, in recent years, science fiction films in European and American countries are still loved by audiences at home and abroad, such as The Martian and Interstellar, which have both commercial and artistic values. It can be seen that the cultural communication of western science fiction movies is more successful than that of China. Taking the above three works as examples, this paper analyzes the doomsday plot, the beauty of returning home and the role shaping of scientific women in science fiction movies from the perspective of the organic combination of "hard-core elements of science fiction" and "soft value in humanity", in an attempt to help the foreign cultural communication of domestic science fiction movies. As an attempt to facilitate the global development of Chinese science fiction, this paper concludes that certain Chinese traditional cultural spirit needs further spreading, that Chinese science fiction and humanity should be combined in a more natural way, and that in particular, female character need in depth and multi-dimensional interpretation.

\section{Introduction}

In the era of vigorous development of science and technology, science fiction carries people's reverie of the unknown, especially the whimsy of exploring the vast universe. As the carrier of this artistic element, science fiction movies are still popular with audiences. Wandering Earth, released in 2019, opens the first year of Chinese science fiction movies, and tells the story of selfhelp when human beings face the earth crisis. Similarly, the themes of crisis and human feelings are also involved in Interstellar released in 2014 and The Martian released in 2015. Why can Interstellar and The Martian successfully spread across cultures from the perspective of western countries?

\section{Doomsday Dilemma Caused by Scientific and Aesthetics Elements}

2. 1 Humanity and Al Technology in Wandering Earth

Some scholars believe that the success of Wandering Earth is largely due to Liu Cixin's novel itself. However, there are many details in the film that are not clearly explained. In fact, what attracts the audience more is the immersive visual experience. Although the plot of Wandering Earth cannot fully meet the expectations of those who favor the novel, it does not hide its flaws. The film depicts sci-fi scenes such as the end of the earth and the underground city in detail, and the visual shock brought by digital special effects makes up for some shortcomings. Moreover, after the film was adapted, it almost only borrowed this setting of the Wandering Earth plan, which is not only the most subtle ingenuity in the original work, but also the most spectacular part of the film: on the one hand, it has a strong Chinese feature that hometown is irreplaceable; on the other hand, only the jet engine flame can create a strong sense of science fiction eschatology. This allows the film to avoid the problem of stacking special effects in domestic sci-fi movies before, but does not polish special effects, so that the story can be kept at the same level as the picture (Li, 2019).

K. C AL-KINDI CENTER R D FOR RESEARCH AN Your gateway to world-class research

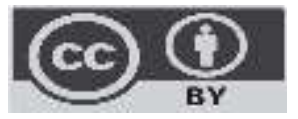

Published by Al-Kindi Center for Research and Development. Copyright (c) the author(s). This open access article is distributed under a Creative Commons Attribution (CC-BY) 4.0 license 
In addition to the special effect, other sci-fi elements are added to the film, such as Al and computers with super computing power. In the film. The Al Moss repeatedly prevented Liu Peiqiang from saving the earth's human beings and even killed the Russian astronaut Lao Ma indirectly. Liu Peiqiang not only discovered that the low-power mode of Moss was just a scorpion, but also pointed out sharply that "Moss, you are murdering". In addition, when Li Yiyi put forward the final plan, but the computer thought that the success probability of the plan was zero, the Coalition Government rejected the plan, and Liu Peiqiang exchanged heroic sacrifice for the success of the Wandering Earth plan, which was also the most powerful counterattack against the authority of $\mathrm{Al}$ and reversed the seemingly doomed human extinction and the so-called Kindling Plan. Therefore, in Wandering Earth, it is still believed that Al may indirectly lead to the destruction of human beings, and that the science and technology represented by human beings and Al oppose each other, ending in the success of human civilization resistance.

\subsection{Human Fighting Natural Disasters in The Martian}

The Martian centers on the story of botanist Mark using scientific knowledge to survive on Mars, which contains the hardest science fiction elements among the three works. Producing oxygen, cultivating potatoes, calculating and distributing available food, and making simple rockets on one's own are rigorous and meticulous, most of which are close to daily life, fully embodying the scientific spirit of astronauts. However, unlike the plight of human extinction in Wandering Earth, The Martian instead centers the doomsday crisis on a single individual, which reflects the sense of insignificance amid the vast universe and the sense of powerlessness when facing the fragility of human beings, thus forming a strong contrast, which is hard for the seemingly powerful scientific knowledge to compete with. In the film, botany knowledge brought a glimmer of life to Mark, but after working hard to create all the basic conditions suitable for survival, a hurricane on Mars wiped out all his efforts; Mark, when finally got in touch with the earth again after twists and turns, was ecstatic, but the team members were faced with the choice of life and death in order to rescue him; When Mark was desperate to get on the convertible rocket and was under an extreme physical condition, Colonel Lewis temporarily decided to stretch out his hand and hold Mark floating in outer space. At the same time, the film chose many overhead shooting angles and far shots. Mark was alone on the vast Mars, looking thin and small, but he tried to survive with his scientific knowledge and strong desire to be alive, which is a tribute to human vitality.

Worth noticing, this film does not blindly advocate the greatness of scientific knowledge. On the contrary, when the team rescued Mark, Beth Johansen, a computational genius, found that the rescue distance measured by the computer was too long, and the plan was difficult to succeed. However, Colonel Lewis left the cabin in spite of himself and completed the impossible task, breaking the prophecy of the computer. At the end of the film, astronauts from many countries cooperated to explore the universe, and Mark's extreme predicament on Mars promoted the space career of human beings on earth. Therefore, in The Martian, people and the unknown world are not antagonistic, but complement each other. In the process of the continuous exploration, human beings encounter natural forces that are difficult to compete with, and exert subjective initiative through scientific knowledge to change and create new things. Instead, they can gain new knowledge and new strength from the crisis.

Although the film focuses more on the individual sense of doomsday, Mark's successful return does not give people the impression of "individual heroism", on the contrary, it embodies the spiritual thought that "man will conquer nature". First of all, because Mark is facing a vast universe, he is called an explorer rather than a hero; Secondly, if Mark didn't have the cooperation of the whole earth and his team, it would be useless for him to work so hard as an individual. Therefore, even if the rescue leader is NASA, there is no obvious individualism tendency, and it is more easily accepted by Chinese culture which emphasizes collectivism.

\subsection{Dynamic Balance between Life and Space-time in Interstellar}

In contrast, the elements of hard science fiction in Interstellar are more abstract and complex, and the dimensions of artistic aesthetics are also richer. The film does not depict too many high-tech sci-fi equipment, but utilizes time as the medium, which changes people's cognition of space and has a subversive interpretation of life: the hero Cooper will hardly have physical aging when living in the outer space, but at the same time, the passage time on the earth is ceased. When he meets his daughter again, her daughter is already skinny and white-haired. 124 years old as he is, he still appears much younger than his daughter and has to accept the fact that her death will be prior to his, and time has created a new dilemma that human beings cannot change. Nonetheless, the mission of human life has not stopped moving forward. Even though her daughter Murphy has grown old, she insists on asking her father to find Professor Brand, who is still in the outer space, and build a new home for mankind together with her. Since then, the love between father and daughter has changed into more selfless love that can be generalized for all mankind. Therefore, the time dilemma in Interstellar has not been overcome by human subjective initiative, but human beings compete with time and space with the simplest form of living, reproduction and inheritance. Only in this way can humans prove their existence in the vast world. 
Some criticize the theme of selfless love to be outdated. Nevertheless, unlike Wandering Earth, in this film human beings have not tried to change the crisis itself, but returned back to nature, continuing human civilization in the most primitive way and resisting the unknowable power of nature with great vitality, thus achieving a delicate dynamic balance.

Comparing the three works, we can find that hard science fiction is also delicately combined with artistic aesthetics, and it is presented with different perspectives: Wandering Earth emphasizes that human beings can overcome the doomsday and the curse from computers, and contrast the beauty of human nature with the evil of $\mathrm{Al}$; The Martian focuses on the dilemma of individuals, focusing on the use of scientific knowledge to actively explore and challenge the unknown, and the final rescue link shows collectivism; Interstellar subverts the fundamental definition of scientific concepts. Although human beings are unable to change their predicament, they still have vitality, which further sublimates and praises the endless power of human civilization.

\section{Core Cultural Values Conveyed through Self-consistent Logic and Humanity}

People's desire for aesthetics comes from two forms of art coexisting, the first is story, the second is lyrical (Jia, 2019). Sci-fi film and television works focus on designing cool special effects and accurately depicting sci-fi imagination, and should also take into account the logical consistency of plot and the smooth expression of humanistic feelings, so as to win a good reputation with good stories. Through different perspectives, the three works discussed in this paper convey different cultural connotations on the beauty of returning home and the shaping of scientific women roles.

\subsection{Beauty of Returning Hometown Interpreted the from Various Perspectives}

The concept of going home appears frequently in Wandering Earth: when Liu Qi fled the underground city with his sister, he asked his sister to go home by car; When Han Ziang visited the detained children, he also emphasized taking them home. These plots express the literal meaning of going home, that is, returning to the residence in the underground city. For astronaut Liu Peiqiang, he can only go home to reunite with his relatives after retirement, and the meaning of home is extended to the Earth. When Russian astronaut Lao Ma encounters an emergency in space and Tim, a Chinese-Australian hybrid in a runaway car, both of them cried out, "Mom, I want to go home", craving for a sense of security in times of crisis. For Han Zi 'ang, the meaning of home is richer: when he sees Shanghai, where the ground is frozen, he tells the stories of the past to the younger generation, but the warm home as an entity no longer exists, only remains in memory; when he died while transporting flint, Han Duoduo said that "grandpa is gone, where lies our home", and the spiritual home collapsed. The disillusionment of these two forms of home brought about the growth of the hero and began to seek the spiritual destination, and the meaning of home was sublimated again. At the most critical moment of the earth, Liu Qi and his rescue team comes to the Forever Night Zone and sees a huge whale, which comes a long way but is frozen. Captain Chloe Wang interprets this as: the whale also wants to go home because returning is the instinct of life. In the end, human attachment to home brings courage to overcome difficulties and compete with the doomsday crisis. The foothold of home lies in the community of human destiny, that is, the guard of all mankind on the earth. It can be seen that Wandering Earth interprets and sublimates the meaning of going home from many angles, and shows a strong will to find a home in various forms.

In The Martian, Mark, who was left behind on Mars, only wanted to return to Earth, and the whole story revolves around saving Mark to go home. At the beginning of the story, NASA wanted to give up the rescue and sacrifice him to take care of the overall situation, but finally chose to carry out the rescue at all costs. From this point, it is not difficult to see that the desire for going home is an emotion with universal human nature. It is precisely because people need to have a home to return to, can they understand that Mark is inhuman to be alone and abandoned by the whole earth, and can have the development of the followup story.

Compared with Wandering Earth, Interstellar describes homecoming in a more detailed way. The film not only highlights people's reluctance to give up the earth and strive to guard their homeland, but also enriches the connotation of homecoming through a series of behaviors of Professor Mann. Like everyone else, Professor Mann is eager to return to the earth. At this time, he already knows that the Earth is about to be destroyed, but he does not hesitate to forge data and harm his companions to get a spaceship that may take him home, and eventually he will die. Different from the previous two works, the interpretation of going home in Interstellar is not so bright, but cast a shadow of humanity. It looks at people's eagerness to go home from another angle, showing the moral dilemma and the dark side of humanity.

\subsection{Scientific Female Roles Shaped with Multi-images}

It is worth noting that both sci-fi works have created full images of scientific women, breaking through the tradition that science fiction films are dominated by men. In The Martian, Colonel Lewis, as the captain, showed extraordinary talents and leadership, and the characters did not lose the elegance and femininity of women. When she was trying to rescue Mark, she jumped out of the flight cabin and was heroic; Beth Johansen, one of the team members, also has a distinct personality. She loves sports, is petite, and is good at computers. Even other male members need her help to check email messages, and her powerful 
computing ability is indispensable to rescue Mark. These designs show that women can become leaders in science as well as men.

However, the female images in Interstellar are slightly complicated. Both Murphy and young Professor Brand were influenced by their fathers, who loved science since childhood and engaged in aerospace work when they grew up. Although they are not as independent as the women in The Martian, the two women play the image of savior in the film, and give full play to the sensitivity and sensibility of women. Daughter Murphy always believes in the existence of ghost, intuitively feels that someone is telling her important things, and finally comes true one by one; Although the young Professor Brand insisted on searching for the black box of the spacecraft because of an emotional incident, resulting in the death of a member of the crew, she realized the power of love and was even willing to gamble intuitively in the unknown universe, thus finding the surviving Professor Mann. Compared with the two, The Martian shows more femininity in appearance, while the images of STEM women in other aspects seem to have little difference with men in traditional science fiction works; but Interstellar affirms femininity itself, and even the sixth sense of women, which has little scientific basis, becomes the key to success. These two perspectives provide a good reference for the portrayal of scientific women in film and television works, especially hard science works.

It seems that the portrayal of female characters in Wandering Earth is not very successful, and the protagonist's growing experience from never knowing the world to saving the Earth is not natural enough, and the contrast before and after is slightly straightforward and abrupt. For example, Han Duoduo was pampered and rebellious at first, and disdained what the squad leader said, Hope is just like DIA, but in the end, she suddenly tried to persuade rescue teams all over the world to act together with this line. Her psychological changes and cognitive understanding of Hope lacked detailed description and coherent mental journey in the film, and she almost completely relied on her brother Liu Qi's shelter during the whole growth process, lacking the ability to be independent. The film is also lacking in the character design of scientific women, and it seems that it still stays at the stage where scientific men dominate. The only scientific female character in the whole film is Zhou Qian, a member of the rescue team. She shot and destroyed flint in order to prevent the members from dying, which led to the stranded rescue plan of the whole team. This impulsive decision seems to be similar to Brand's perceptual intuition in Interstellar, but Zhou Qian's handling is simple, rude and emotional, and she lacks effective communication with the players. Although her judgment is reasonable, it can't make the audience feel the approval of any femininity. In the absence of foreshadowing, Zhou Qian roared and rationally destroy the rock that saved mankind. On the contrary, it seemed that other male players attached great importance to their feelings and were desperate. Generally speaking, the moments when the female images have the most sense of existence in the film happen to be when they are hysterical. Regardless of whether Zhou Qian, who has been entrusted with a heavy responsibility, will be so impulsive as a well-trained rescue worker, if the film wants to highlight the femininity of these characters, it may be possible to use more delicate and subtle expressions instead of letting off steam to express inner strength.

The flattening of female roles may be one of the shortcomings of the cross-cultural communication of Wandering Earth. Western values emphasize the independent spirit of individuals, especially the self-sustaining of women, so they prefer independent and powerful female roles. In the comment area of IMDB film review website, there are also many foreign audiences who say that some roles of Wandering Earth are unreasonable. The extensive expression of the leading girl's psychological growth and the flattening or even lack of the role of scientific women are probably one of the reasons for the unsuccessful cross-cultural communication.

\section{Soft and Hard Power Strengthen in Cultural Communication and Core Competitiveness}

As a phenomenal film, Wandering Earth performed well in China, but its response in overseas markets was flat, which revealed that domestic sci-fi films should take into account the cultural core of spreading Chinese-style hard sci-fi in the future, while reducing some flaws in terms of film plot, logic and role modeling.

At present, the main difficulties in spreading culture through Wandering Earth lie in that, on the one hand, many cultural spirits are not popular enough, such as collective heroism, community of human destiny and other concepts, Chinese family ethics, Chinese dialects, etc., which are not deeply rooted in the hearts of the people. Therefore, many viewers cannot understand certain symbols, and when the film and television works do not explicitly interpret these cultural images thoroughly, the viewers will be confused, especially the western audience; on the other hand, the insufficient description of the details, the failure to create a cultural atmosphere and sense of substitution, and too many elements together make the audience feel overwhelmed and unable to empathize.

It can be seen from IMDB's film review that the audience thinks that the time setting of the story is not far away from now, but the scene design in the film is quite out of touch with modern times. In addition, there are many details that lack description, such as how to build underground cities, how to transfer human beings, why the value of dried earthworms suddenly rises instead of other more daily foods, what the air pressure on the surface is, and how to use flints, etc. Especially for the audience 
who have never read the original works, the explanations of these important backgrounds are not clear enough. In addition, the logic of many plots is not strict or redundant. For example, the planning and arrangement of the rescue team is not clear enough, and it is even inferior to the modern public security system; Al can calculate the success rate of all schemes, but when some thrusters fail, there is no complete emergency plan in the world, and only the old-fashioned broadcast directly advises everyone to give up. Even the machine can calculate that the Earth can last for seven days, which is inconsistent with the figure estimated by technician Li Yiyi. There are still many gaps in details, such as why Liu Qi became registered permanent residence and Li Yiyi became Li Changtao. Is this showing children's naivety or trying to be humorous? When the audience sees these lines, they will stop instinctively. On a certain level, vague explanations disconnection the audience and the movie, impairing the viewing experience. Admittedly, it is not easy to explain such a huge story structure and complicated details within the time limit of a movie, but eliminating the obvious logic loops is the prerequisite for telling a good story. If the core of culture is not popular enough and if the expression of movies is blunt, hard science and soft humanities cannot be compatible, and therefore it will worsen the cultural communication.

\section{Conclusion}

The heated discussion of Wandering Earth has passed, but with the rapid development of science and technology, the discussion of Chinese science fiction movies continues. While learning from Hollywood and other western science fiction films, China should also foster Chinese characteristics. The effective combination of hard science fiction and soft humanity sublimates the main idea, interprets the relationship among man, nature and future science and technology from multiple dimensions, continues to explore the cultural spirit with Chinese characteristics, such as the beauty of returning home and the spirit of foolish old people, and creates vivid and three-dimensional images of scientific women, so that science fiction works with oriental characteristics can be widely disseminated and recognized. Future research could lay more emphasis, from critical perspectives, on the effective combination of humanity and science fiction, more acceptable means to convey cultural values and multi-dimensional interpretation of female characters in order to facilitate the development and popularization of Chinese science fiction.

Acknowledgments: This paper is part of the achievement of the University Students Innovation Project of Shanghai in 2020, "Nationality and Cosmopolitanism of Film and Television Literature and its Cross-cultural Communication in the Context of Globalization" (S20107)

Conflicts of Interest: The authors declare no conflict of interest. "The funders had no role in the design of the study; in the collection, analyses, or interpretation of data; in the writing of the manuscript, or in the decision to publish the results".

\section{References}

[1] Jiang S., Chen L. (2020). Based on the Connotation of Contemporary Sci-fi Movies Talking About Marxist Scientific and Technological Aesthetics with Chinese Characteristics in the New Era [J]. Chinese film market, (11),52-55.

[2] Tian S. (2020). Pandora as Gaia: Study on the natural view of Avatar [J]. Dialectics of Nature Communication, 42(11),37-43.

[3] Liu T. (2020), Localization strategy of domestic sci-fi films [J]. Contemporary Film, (10),129-134.

[4] Pan Y. (2020), Research on American Film Criticism in 2019 [J]. World Film, (05), 149-178.

[5] Ma E., Chen J.(2020), Dilemma and Development of Domestic Science Fiction Films since the Founding of New China [J]. Film Literature, (19), 34-38.

[6] Lu Z., Zhao Y.(2020), Body Meaning in Digital Age: An Analysis of Three Virtual Human Body Paradigms in Science Fiction Movies [J]. Journal of Fujian Normal University (Philosophy and Social Sciences Edition), (05), 116-124-171.

[7] Chen X. (2020), Analysis on the Regression Proposition of Contemporary Space Films [J]. Film Literature, (08), 65-67.

[8] Chen Y(2019). The way to open the first year of Chinese science fiction: the space political aesthetics of Wandering Earth and the expression of Chinese science fiction concept [J]. New Film, (02), 97-102.

[9] Jiang Z.(2019) The formation of science fiction "soft and hard" and its influence and limitations in China [J]. Chinese Literary Criticism, (04), 149-156-160.

[10] Gai Q.(2019), The new world order in the doomsday era: Wandering Earth and the narration of a new big country [J]. Exploration and Contention, (03), 73-80

[11] Chen X, Yang C., Li N., Gao Y.(2015), Interstellar and Science Fiction Film: Types, Narrative and Cultural Spirit [J]. Creation and Review, (16), $112-128$.

[12] Chu H.(2017), Science Fiction and Truth: An Analysis of the Multiple Aesthetic Cultures of the Film "The Martian" [J]. Film Review, (17),52-54.

[13] Zhao J.(2017), The Martian: Self-redemption of Lonely People [J]. Film Literature, (05), 141-143. 